\title{
A novel neurodevelopmental disorder associated with compound heterozygous variants in the huntingtin gene
}

\author{
This paper has been amended since online publication and a corrigenda also appears in this issue
}

\author{
Lance H Rodan ${ }^{\star, 1}$, Julie Cohen ${ }^{2}$, Ali Fatemi², Tammy Gillis ${ }^{3}$, Diane Lucente ${ }^{3}$, James Gusella ${ }^{3,4}$ and \\ Jonathan D Picker ${ }^{1}$
}

\begin{abstract}
We report compound heterozygous variants in $H T T$, the gene encoding huntingtin, in association with an autosomal recessive neurodevelopmental disorder. Three siblings presented with severe global developmental delay since birth, central hypotonia progressing to spastic quadraparesis, feeding difficulties, dystonia (2/3 sibs), prominent midline stereotypies (2/3), bruxism (1/3), high myopia (2/3), and epilepsy (1/3). Whole exome sequencing identified compound heterozygous variants in $H T T$ that co-segregated in the three affected sibs and were absent in an unaffected sib. There were no additional variants in other genes that could account for the reported phenotype. Molecular analysis of HTT should be considered, not just for Huntington's disease, but also in children with a Rett-like syndrome who test negative for known Rett and Rett-like syndrome genes. European Journal of Human Genetics (2016) 24, 1826-1827; doi:10.1038/ejhg.2016.74; published online 22 June 2016
\end{abstract}

\section{INTRODUCTION}

Huntington's disease (HD) is a devastating neurodegenerative disorder caused by trinucleotide repeat expansion in HTT, the gene encoding huntingtin. ${ }^{1}$ We present siblings affected by a severe, congenital neurodevelopmental disorder associated with central hypotonia, spastic quadraparesis, and variable dystonia, midline stereotypies, epilepsy, and high myopia associated with compound heterozygous variants predicted to reduce huntingtin function.

\section{SUBJECTS}

The family was composed of healthy parents, a healthy and developmentally normal 3-year-old boy, and three affected children described below. Family ancestry was from Ecuador.

Sibling 1 is a 12 -year-old girl presenting with developmental delay and abnormal tone. She was the product of an uncomplicated, term pregnancy. She was globally hypotonic since birth with weak sucking and poor feeding. Her development was severely globally delayed. From 1 year, she developed progressive appendicular hypertonia with intermittent dystonic posturing. L-dopa provided no clinical benefit. She also has midline stereotypies including hand wringing. She has never had seizures. She is currently able to briefly sit unsupported, and uses a few words. She is being evaluated for dysphagia. She also has severe myopia ( -9 diopters).

Examination noted relative macrocephaly (98th percentile), height at second percentile, and weight at first percentile. She had saccadic ocular pursuit. She was spastic in all four limbs with generalized hyperreflexia and sustained ankle clonus. She had distal limb dystonia; both wrists were held in a flexed posture with hyperextended metacarpophalangeal joints, and halluces were spontaneously extended (striatal toe sign). She appeared mildly bradykinetic.

MRI of brain with spectroscopy was normal at ages 9 months, 4 and 8 years. Laboratory investigations were normal including karyotype,
MECP2 sequencing and deletion/duplication testing, CDKL5 sequencing, CSF neurotransmitters, CSF glucose, leukocyte lysosomal enzymes, carbohydrate deficient transferrin, plasma amino acids, urine organic acids, lactate, guanidinoacetate, 7-dehydrocholesterol, and very long chain fatty acids.

Sibling 2 is a male born at term following an uncomplicated pregnancy. He was noted to be hypotonic since birth. Developmental milestones were globally delayed. He babbled at 8 months of age, but never acquired spoken language. He demonstrated frequent midline hand-wringing stereotypies and bruxism. He had chronic feeding difficulties, gastroesophageal reflux, and oromotor dysfunction leading to placement of a G-tube at age 3 years. He had high myopia ( -9 OD and -8 OS). He developed seizures at age 4 years. EEG demonstrated slowing, spike-and-slow-wave complexes, and sharp waves.

At his last evaluation at age 6 years, weight was 58th percentile, height was seventh percentile, and head circumference was 50th percentile. He was unable to sit unsupported, unable to follow commands, and had no spoken words, but was socially interactive. He had severe truncal hypotonia, poor head control, spastic quadriplegia, contractures, and dystonic posturing of lower extremities.

This child died unexpectedly at age 7 years from respiratory distress of undetermined cause. No postmortem evaluation was performed.

MRI of brain at age 1 year was normal. BAC array comparative genomic hybridization was normal. Muscle biopsy of left quadriceps showed normal histology and immunostaining.

Sibling 3 is a 2-year-old male. Decreased fetal movements were noted prenatally. He was born at term. He was severely hypotonic since birth. Poor feeding necessitated gastrostomy tube placement. He has severe developmental delay without regression. Currently, he has not achieved head control. He can reach, but cannot hand across midline. He has no vocalizations or receptive language. History notes

${ }^{1}$ Division of Genetics and Genomics, Boston Children's Hospital, Boston, MA, USA; ${ }^{2}$ Division of Neurogenetics, Kennedy Krieger Institute, Johns Hopkins Medical Institutions, Baltimore, MD, USA; ${ }^{3}$ Center for Human Genetic Research, Massachusetts General Hospital, Boston, MA, USA; ${ }^{4}$ Department of Genetics, Harvard Medical School, Boston, MA, USA *Correspondence: Dr LH Rodan, Division of Genetics and Genomics, Boston Children's Hospital, 300 Longwood Avenue, Boston, MA 02115 , USA. Tel: +1 617 877 2918; Fax: +1 617730 0466; E-mail: Lancerodan@gmail.com

Received 5 March 2016; revised 28 April 2016; accepted 26 May 2016; published online 22 June 2016 
gastroesophageal reflux, recurrent pneumonias, and strabismus. $\mathrm{He}$ does not have myopia.

Examination demonstrated normocephaly (58th percentile), with length at 41st percentile and weight at 38th percentile. Extraocular movements appeared full. He had axial hypotonia and appendicular hypertonia. His feet were fixed in an equinovarus position.

Neuroimaging was declined by parents.

\section{RESULTS}

Family exome sequencing demonstrated all affected children to be compound heterozygotes for variants in HTT (c.[4469+1G>A]; $[8156 \mathrm{~T}>\mathrm{A}])$. These variants were confirmed by Sanger sequencing in the affected daughter. The unaffected 3.5-year-old brother carried neither variant. The paternally inherited variant, c. $[4469+1 G>A]$, destroys a canonical splice donor site in intron 34 and is predicted to result in abnormal gene splicing. The maternally inherited p.(F2719L) (c.[8156T > A]; NM_002111.7; NG_009378.1) variant occurs at a highly conserved site. In silico analysis with Polyphen2 and Mutation Taster both predict this variant as damaging to the protein structure and function with a probability $>0.99$. Neither variant has been observed in $~ 6000$ individuals of European and African American ancestry by the NHLBI exome sequencing project nor by the Exome Aggregation Consortium (ExAC). The exome did not reveal any additional homozygous, compound heterozygous, or de novo variants that could account for the siblings' phenotype. The mean depth of coverage was $117 \times$, and at least $96.8 \%$ of the exome was covered at a minimum of $10 \times$. The reported variants were submitted to the ClinVar database (http://www.ncbi.nlm.nih.gov/clinvar/) with accession numbers SCV000267153 (HTT NM_002111.7:c.8157T > A) and SCV000267152 (HTT NM_002111.7:c.4469+1G>A).

\section{DISCUSSION}

We report three siblings with a novel neurodevelopmental disorder associated with static global developmental delay since birth, central hypotonia progressing to spastic quadraparesis, and feeding difficulties. Two of the three siblings additionally had features suggestive of a Rett-like syndrome. All three siblings had compound heterozygous variants in HTT on whole exome sequencing, with no additional biallelic or de novo variants in disease-related genes to account for their phenotype. The exome platform had adequate depth of coverage.

$\mathrm{HD}$ is an autosomal dominant neurodegenerative disorder associated with dementia and extrapyramidal symptoms. HD typically presents in middle age, although rarely in childhood and exceptionally in infancy. HD is caused by a CAG trinucleotide expansion in the first exon of HTT, lengthening a polyglutamine tract in the mutant huntingtin protein. Genetic analyses of human HD and of precise genetic models in the mouse indicate that the disorder is due to a completely dominant gain-of-function acting through some normal aspect of the structure, function, or localization of huntingtin., ${ }^{1,2}$

Mice heterozygous for null variant of $\mathrm{Htt}$, the murine ortholog of $H T T$, are phenotypically normal. However, homozygous $H t t$ knockout mice do not survive past the embryonic gastrula stage, indicating that some level of contribution of huntingtin is essential. ${ }^{3}$ Markedly reduced huntingtin levels from hypomorphic $\mathrm{Htt}$ alleles have implicated huntingtin in neurogenesis and result in reduced body size, movement abnormalities, and a variable increase in ventricle volume. ${ }^{4,5}$ If the mouse requirement for at least a low level of huntingtin for viability is reflected in the human, we would expect to find reduced function rather than complete inactivation of huntingtin associated with postnatal developmental phenotypes.

Heterozygous inactivation of HTT due to its interruption by a balanced translocation has been reported in two related adult individuals with no abnormal phenotype, indicating that a single HTT allele is sufficient to support normal development. ${ }^{6}$ Notably, another group recently reported an 18-year-old woman with a Rett syndrome phenotype associated with compound heterozygous variants in HTT detected on whole exome sequencing. ${ }^{7}$

The ExAC database lists HTT as a highly constrained gene (probability of loss-of-function intolerance or $\mathrm{pLI}=1.00$ ), in that it exhibits far fewer loss-of-function variants in the population than expected, suggesting that such alleles are at a strong selective disadvantage. Still, ExAC reports 23 individuals who are homozygous for a termination codon in exon 1 of HTT (p.(Gln25Ter)). If accepted at face value, this variant would certainly argue that loss of function does not generate a severe phenotype, which would be at odds with the murine and human population data; however, there are some reasons to at least be doubtful concerning the existence of this variant and its truncating nature. This variant lies in a region that has very low coverage and low complexity. None of the alleles has been Sanger confirmed. Allele calling in such a region is fraught with error, indels are frequent, and the calls in this instance were on the low side of the quality score distribution. Perhaps most concerning is that 23 of 28 individuals with this allele are homozygotes, a massive and problematic deviation from Hardy-Weinberg equilibrium.

Of particular interest is the resemblance of phenotype in $2 / 3$ of our reported sibs to a Rett-like syndrome, and the independent report of a Rett syndrome phenotype in another individual with biallelic variants in HTT. A possible explanation for this overlap in phenotype may lie in the direct interaction of huntingtin with methyl-CpG-binding protein 2 (MeCP2), the protein implicated in classic Rett syndrome, as demonstrated in mouse and cellular models of HD. ${ }^{8}$ Sequencing of HTT could be considered in the evaluation of the child with a Rett-like syndrome who tests negative for MECP2 and the other well established Rett-like genes. Additional cases with HTT pathogenic variants and functional studies will be required to confirm our findings and to further define phenotype of this huntingtin-deficiency disorder.

\section{CONFLICT OF INTEREST}

The authors declare no conflict of interest.

1 Gusella JF, MacDonald ME: Huntington's disease: seeing the pathogenic process through a genetic lens. Trends Biochem Sci 2006; 31: 533-540.

2 Lee JM, Ramos EM, Lee JH et al: CAG repeat expansion in Huntington disease determines age at onset in a fully dominant fashion. Neurology 2012; 78: 690-695.

3 Duyao MP, Auerbach AB, Ryan A et al: Inactivation of the mouse Huntington's disease gene homolog Hdh. Science 1995; 269: 407-410.

4 White JK, Auerbach W, Duyao MP et al: Huntingtin is required for neurogenesis and is not impaired by the Huntington's disease CAG expansion. Nat Genet 1997; 17: 404-410.

5 Auerbach W, Hurlbert MS, Hilditch-Maguire P et al: The HD mutation causes progressive lethal neurological disease in mice expressing reduced levels of huntingtin. Hum Mol Genet 2001; 10: 2515-2523.

6 Ambrose CM, Duyao MP, Barnes G et al: Structure and expression of the Huntington's disease gene: evidence against simple inactivation due to an expanded CAG repeat. Somat Cell Mol Genet 1994; 20: 27-38.

7 Lopes F, Barbosa M, Ameur A et al: Identification of novel genetic causes of Rett-syndrome like phenotypes. J Med Genet 2016; 53: 190-199.

8 McFarland KN, Huizenga MN, Darnel SB et al: MeCP2: a novel Huntingtin interactor. Hum Mol Genet 2014; 23: 1036-1044. 